\title{
BMJ Open Prospective cohort study examining cervical cancer screening methods in HIV-positive and HIV-negative Cambodian Women: a comparison of human papilloma virus testing, visualization with acetic acid and digital colposcopy
}

\author{
Sovannara Thay, ${ }^{1}$ Andrew Goldstein, ${ }^{\circ 2,3}$ Lena Sophia Goldstein, ${ }^{4}$ \\ Vaishnavi Govind, ${ }^{3}$ Kruy Lim, ${ }^{1}$ Chanthou Seang ${ }^{1}$
}

To cite: Thay S, Goldstein A, Goldstein LS, et al. Prospective cohort study examining cervical cancer screening methods in HIV-positive and HIVnegative Cambodian Women: a comparison of human papilloma virus testing, visualization with acetic acid and digital colposcopy. BMJ Open 2019;9:e026887. doi:10.1136/ bmjopen-2018-026887

- Prepublication history for this paper is available online. To view these files, please visit the journal online (http://dx.doi. org/10.1136/bmjopen-2018026887).

Received 2 0ctober 2018 Revised 19 December 2018 Accepted 17 January 2019

Check for updates

(C) Author(s) (or their employer(s)) 2019. Re-use permitted under CC BY-NC. No commercial re-use. See rights and permissions. Published by BMJ.

For numbered affiliations see end of article.

Correspondence to Dr Andrew Goldstein; obstetrics@yahoo.com

\section{ABSTRACT}

Objectives Logistical and economic issues make traditional cytology-based cervical cancer screening challenging in developing countries. Alternative, costeffective, screening strategies must be developed to screen millions of women in resource-poor countries such as Cambodia.

Design A prospective cohort study during which all women underwent four cervical cancer screening methods: (1) self-sampled human papilloma virus (HPV) testing (careHPV system), (2) clinician-collected HPV testing, (3) visualization with acetic acid (VIA) and (4) digital colposcopy (DC) with the Enhanced Visual Assessment System (EVA).

Setting A referral hospital in Phnom Penh, Cambodia. Participants Two hundred and fifty Cambodian women (129 HIV+, 121 HIV-). Subjects were recruited from the National Center for HIV/AIDS Dermatology and sexually transmitted disease (STD) cohort, the Sihanouk Hospital Center of Hope's Rural Outreach Teams and the Pochentong Medical Center.

Results Fifty six of the 250 (22.4\%) patients tested positive for high-risk HPV (hrHPV+). Thirty seven of the 129 HIV+ women were hrHPV+ (28.6\%) whereas 19/121 HIV- women were hrHPV+ $(15.7 \%) p=0.0154$. Selfsampling HPV specimens identified 50/56 (89\%) whereas physician-collected specimens identified $45 / 56$ (80\%) $p=0.174 .95 .2 \%$ of the patients felt comfortable obtaining HPV self-samples. Thirty seven of 250 women were VIA+. Thirty of $37 \mathrm{VIA}+$ women underwent confirmatory biopsies for cervical intraepithelial neoplasia (CIN) (26 CIN1, 4 $\mathrm{CIN} 2+)$. The rate of confirmed dysplasia in the HIV+ group was 20/129 (15.5\%) compared with 10/121 (8.26\%) in HIV- women $p=0.0291$. The contemporaneous physician impressions of the DC images accurately differentiated between CIN1 and CIN2+ lesions in all 30 women having confirmatory biopsies.

Conclusions The results of this study suggest potential modifications of the current cervical screening strategy
Strengths and limitations of this study

This prospective cohort study is the first to demonstrate that Cambodian women are comfortable and can adequately perform human papilloma virus (HPV) self-sampling and that rapid, high volume HPV testing with the careHPV system is feasible in Cambodia.

- This study demonstrates that digital colposcopy, using a new, hand-held digital colposcope with advanced optics, is superior to visualis ation with acetic acid as it allows the examiner to differentiate between cervical intraepithelial neoplasia (CIN) 1 and CIN2+ lesions.

- As this cohort study was performed in an urban hospital, the results may not be as applicable to more rural regions of Cambodia.

that is currently being employed in Cambodia. The first step in this new strategy would be self-swabbing for hrHPV. Subsequently, hrHPV+ patients would have DC and immediate treatment based on colposcopic findings: cryotherapy for suspected CIN1 and loop electrosurgical excision procedure (LEEP) for suspected CIN2+.

\section{BACKGROUND}

Cervical cancer is the third most common cancer among women worldwide with an estimated 569847 new cases annually and 311365 deaths. ${ }^{1}$ Due to increased prevention measures in developed countries, up to $85 \%$ of the disease burden lies in developing countries with limited resources for human papilloma virus (HPV) vaccinations and pelvic screening. ${ }^{2}$ In Cambodia, cervical cancer is the leading cancer in females with 1515 
new cases diagnosed each year and 795 deaths. Cervical cancer rates are specifically high in Cambodia due to lack of widespread prevention. In Cambodia, cervical cancer deaths have surpassed maternity-related deaths, making it a leading healthcare priority. ${ }^{3}$

Human papillomavirus (HPV) is the most common sexually transmitted disease (STD) in the world, affecting $15 \%-25 \%$ of all women. ${ }^{4} \mathrm{HPV}$ also accounts for nearly all of the cases of cervical cancer. Infections of HPV strains $6,11,16,18,31,33,45,52$ and 58 are responsible for $90 \%$ of cervical carcinoma. ${ }^{5}$ Primary prevention requires vaccination against HPV before girls become sexually active in order to prevent infection of HPV types included in the vaccine. The vaccine has recently become available at a lower cost, making it accessible in developing nations though not yet widely available in Cambodia. ${ }^{6}$

In congruence with vaccinations, regular screening, or secondary prevention, can greatly diminish the number of cervical cancer cases by treating precancerous lesions earlier. IARC has estimated that if all women aged 35-64 years were screened every 5 years and treated as necessary, the incidence of cervical cancer would be reduced by $84 \% .{ }^{78}$ Unfortunately, few Cambodian women are currently receiving preventative services. ${ }^{6}$

With a HIV prevalence of $0.8 \%$ in Cambodia, there is a specific need to screen HIV-positive women who make up $50 \%$ of adults living with HIV. ${ }^{9}$ Many studies have shown a clear link between HPV infection and cervical dysplasia with HIV-positive status. ${ }^{10}$ HPV infection advances more frequently and quickly in HIV-infected women due to their compromised immune system. This causes cancerous and precancerous lesions to appear earlier after the initial HPV infection than in HIV-negative women. Women with HIV are 4.6 times more likely to develop cervical cancer than uninfected women. ${ }^{11}$ Additionally, the dysplastic lesions are comparatively larger in size and number as well as more likely to reoccur after treatment. ${ }^{12}$ Relative immunity has been shown to be an underlying factor in progression of cervical cancer. Lower CD4 counts have been correlated with aggressive forms of the HPV virus; however, highly active antiretroviral therapy (HAART) may reduce HPV-related disease advancement or severity. ${ }^{13}$

The association of HIV and HPV can be explained by immunosuppression in HIV-infected women, and also by the fact that both HIV and HPV are sexually transmitted infections. Specific risky behaviours that play a role in the transmission of HIV are also applicable to the transmission of HPV. HPV can also be transmitted without penetrative sexual acts as it can be passed through skin-to-skin contact during sexual activity, increasing the probability of transmission compared with HIV.

Due to lack of healthcare resources throughout Cambodia, the traditional multistep strategies of cytology (pap smear), colposcopy, biopsy and subsequent treatment of women diagnosed with cervical dysplasia are not feasible. ${ }^{3}$ As such, less resource intensive screening techniques have been studied to screen for cervical dysplasia and carcinoma. These screening tests include visualisation with acetic acid (VIA) and HPV testing. ${ }^{145}$

The VIA screening technique is an appropriate and effective approach to prevent cervical cancer. VIA uses $3 \%=5 \%$ acetic acid (household vinegar) applied to the cervix to illuminate cervical abnormalities. These lesions are detectable by the naked eye as the acetic acid solution causes them to turn white (acetowhite). Immediate results from direct visualisation enables the women's health specialist to treat appropriate precancerous lesions with cryotherapy in the same visit. ${ }^{16}$ In resource-poor settings, VIA with cryotherapy is a promising 'screen-and-treat' strategy as it does not require expensive equipment and the patient can be treated on the same day, minimising loss to follow-up. However, VIA can be affected by poor visual acuity of the examiner, a dim light source, inadequate training of the examiner, and it produces no permanent image for documentation or quality control.

Cervical HPV DNA testing has been shown to be reliable for the detection of high-grade cervical intraepithelial neoplasia (CIN).${ }^{17}$ Studies have shown that healthcare provider-collected cervical samples have significantly higher sensitivity and only slightly lower specificity for detecting high-grade CIN as compared with traditional cytology ${ }^{18}{ }^{19}$ In addition, the possibility of using self-collected vaginal samples for HPV testing has also been recently explored to increase the number of women screened. ${ }^{15-17}$ The preliminary results of self-collected HPV testing have been promising but have suggested the need for the approach to be investigated in different geographical and demographical settings. The careHPV testing system (QIAGEN, Maryland, USA) is a simple, faster, low-cost, semiportable and robust method for HPV testing, and a new variant of the traditional Hybrid Capture 2 System (HC2) that was designed to work in low-resource settings. ${ }^{17}$ Each careHPV system can run 90 specimens in approximately 3 hours.

Digital colposcopy (DC) is similar to VIA in which the cervix is visualised after the application of acetic acid. In contrast to VIA, DC enables photography of the cervix to allow for magnified visualization of surface morphology. DC also facilitates telemedicine support, and patient and provider education. ${ }^{20} 21$ The Enhanced Visual Assessment System (EVA) (MobileODT.com) (figure 1) utilises the advanced optics found in Android smartphones that are ubiquitous even in low-resource settings. DC may potentially have improved sensitivity and specificity as compared with VIA as the digital image can be greatly magnified. ${ }^{22}$ In addition, DC adds very little to the cost of visualization of the cervix and produces a permanent image that can be used for documentation and quality control. Lastly, the images obtained with DC can be sent electronically to expert consultants to evaluate more difficult cases.

\section{OBJECTIVES}

The goals of this research were to assess the ease at which Cambodian women can obtain self-sampled HPV tests and 


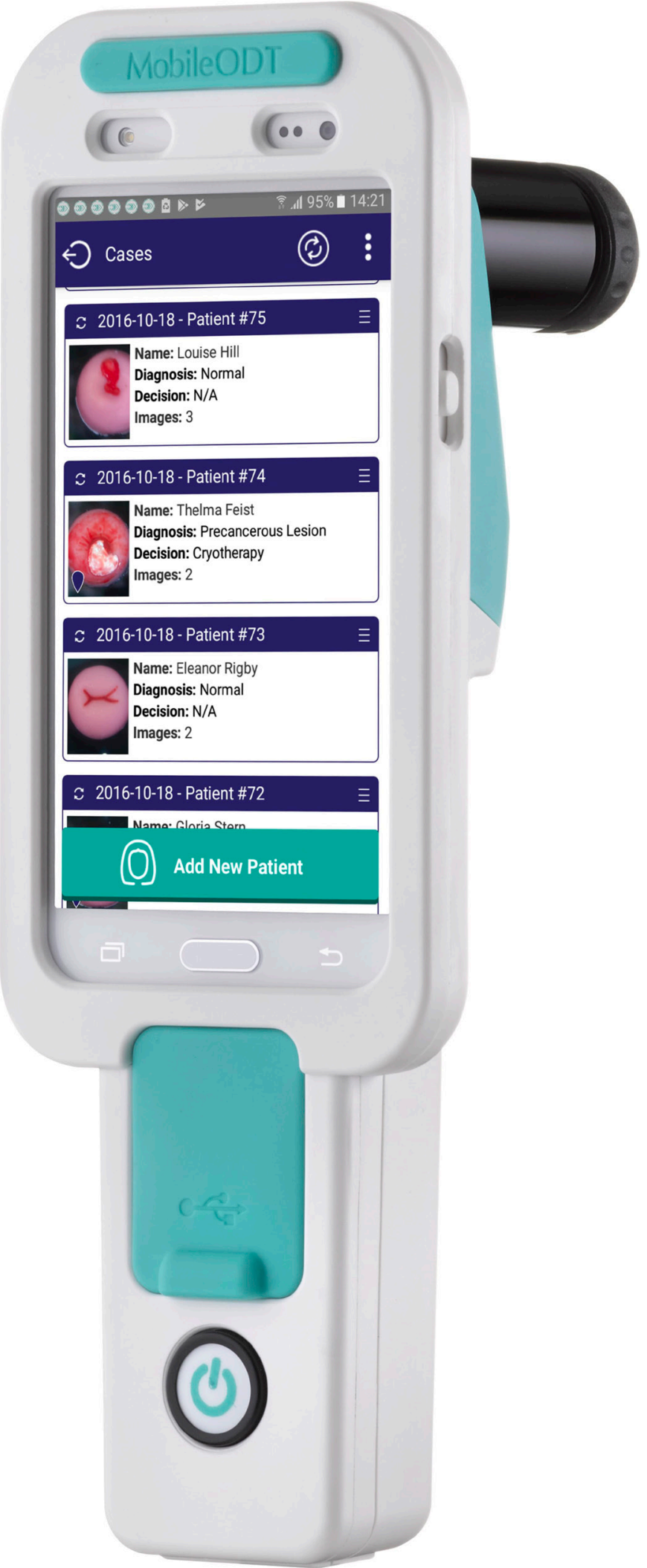

Figure 1 Enhanced Visual Assessment System, MobileODT.

to determine their comfort level in obtaining these samples; to compare the sensitivity of self-sampled HPV testing in Cambodian women as compared with clinician-collected samples; to determine and compare prevalence rates of high-risk HPV infection (hrHPV) and cervical dysplasia in HIV-positive and HIV-negative Cambodian women; and to determine if DC is superior to VIA by improving the providers' ability to distinguish between CIN1, CIN2+ and cervical cancer. The research is intended to help the Cambodian Ministry of Health to design a cost-effective, large-scale, rapid, screen-and-treat cervical cancer programme for Cambodian women.

\section{MATERIALS AND METHODS}

Between 1 October 2016 and 30 September 2017, 250 Cambodian women between 30 and 49 years of age (129 HIV-positive and 121 HIV-negative) were screened at the Sihanouk Center of Hope in Phnom Penh, Cambodia. Subjects were recruited from the National Center for HIV/ AIDS Dermatology and STD cohort, the Sihanouk Hospital Center of Hope's Rural Outreach Teams and the Pochentong Medical Center.

After obtaining informed consent, subjects were instructed on how to obtain a self-sampled vaginal specimen for HPV testing. Then they obtained the sample and filled out a questionnaire that assessed their comfort level in obtaining the self-sample. A cervical examination was then performed with a speculum. The provider obtained another swab for HPV testing. Both swabs were then tested for 14 genotypes of hrHPV $(16,18,31,33,35,39,45,51,52$, $56,58,59,66,68)$ using the careHPV system. Acetic acid was then applied to the cervix and VIA was performed. Thin acetowhite lesions were considered positive and suspicious for CIN1 lesions. Thick acetowhite lesions, lesions with mosaicism or punctation, or abnormal blood vessels were considered positive and suspicious of CIN2+ lesions. An assistant contemporaneously documented all of the provider's findings. The EVA system was then used to obtain DC images (1-3 images per patient). The investigator then examined the DC images to see if these images change the diagnosis made with VIA alone. If either VIA or DC images were positive for cervical abnormalities, patients were triaged to one of two possibilities. (1) Cryotherapy was performed the same day when either VIA or DC images were suspicious for CIN1 lesions. (2) Cervical biopsy was performed when either VIA or DC images were suspicious for CIN2+ dysplasia or cervical cancer. Patients who have biopsy proven advanced disease (CIN2+) returned within a week for loop electrosurgical excision procedure (LEEP).

The specific variables being measured in this study include the following. (1) The percentage of Cambodian women who feel comfortable obtaining self-swabs for hrHPV. (2) The rate of hrHPV positivity in self-sampled swabs and clinician-collected samples. (3) The rates of cervical dysplasia in HIV-positive and the HIV-negative Cambodian women.

\section{Patient and public involvement}

This study was designed to explore new strategies for cervical cancer screening in Cambodian women. The study designers considered issues regarding the burden of screening on the patients. Specifically, the cost to the individual patients, the time needed for screening, the 
burden of positive results, need for further treatment and the risk of being lost to follow-up were all considered. Input from patient advocates including the Sihanouk Hospital Center of Hope's Rural Outreach Teams was obtained during the development of the study design. In addition, patients were specifically asked whether they felt comfortable performing self-swabs for HPV testing and whether they preferred self-swabbing or provider swabbing. All patients were given their results of the HIV tests, HPV tests, the results of VIA, and they were shown their DC images. Lastly, patients were given education and counselling regarding the HIV and HPV prevention and screening for themselves and for their friends and relatives.

\section{Statistical analysis}

Patient questionnaire responses and information from patients' charts were collected and entered into EpiData. The data was analysed using SPSS V.24.0 statistical software. Fischer's exact test of independence was performed to determine if there was an association between hrHPV infection, VIA+ and cervical dysplasia with HIV status. McNemar's test was performed to determine specificity of self-sampling versus physician-obtained specimens in diagnosing hrHPV. P values were two-sided, and statistical significance was defined as $\mathrm{p}<0.05$.

\section{RESULTS}

All 250 women were screened using all four techniques (129 HIV+, 121 HIV-). Fifty six of the 250 (22.4\%) women tested positive for high-risk HPV (hrHPV+). Of these 56 women, 39 tested positive in both self-sampled specimen and physician-obtained specimen. Eleven women were positive only in self-sampling, and six were positive only in the physician-obtained specimens. Self-sampling identified 50/56 (89\%) whereas physician-obtained specimens only identified $45 / 56(80 \%) \mathrm{p}=0.174 \mathrm{NS}$. Thirty seven of the $129 \mathrm{HIV}+$ women were hrHPV+ $(28.6 \%)$ whereas 19/121 HIV- women were hrHPV+ (15.7\%) p=0.0154; 952 of the patients reported that they felt comfortable obtaining the HPV sample. 968 of the patients reported that they were confident to obtain a proper sample on their own. Thirty seven of the 250 women were VIA+ [23/129 (17.8\%) HIV+], [14/121 (11.5\%) HIV-] $\mathrm{p}=0.149$. Thirty of the 37 VIA+ women underwent confirmatory biopsies (26 CIN1, 4 CIN2+), while seven patients refused confirmatory biopsy. The rate of confirmed cervical dysplasia in the HIV+ group was 20/129 $(15.5 \%)$ as compared with only $10 / 121(8.26 \%)$ in HIV- women $p=0.0291$. The contemporaneous provider impressions of the DC images accurately differentiated between CIN2+ and CIN1 lesions in all 30 women who had biopsies.

\section{DISCUSSION}

There are over 8 million women at risk for cervical cancer in Cambodia. In addition, Cambodia has one of the lower rates of physicians per 1000 women in Asia (0.177).$^{23}$ As the vast majority of Cambodian women have not been screened for cervical cancer, screening strategies must be developed to screen-and-treat large numbers of women for cervical dysplasia and cancer. This is especially true for approximately 39000 Cambodian women with HIV disease.

The results of this study might suggest potential modifications of the current cervical screening strategy that is currently being employed in Cambodia on a limited basis. The first component of this strategy would be HPV self-sampling. The results of this study confirm that 95.2\% of Cambodian women are comfortable obtaining these specimens. Previous studies have shown that self-obtained specimens have a higher sensitivity than pap smears in detecting CIN2+ lesions. ${ }^{16}{ }^{17}$ In addition, many studies, including meta-analyses, have shown that self-obtained vaginal swabs are only marginally lower in sensitivity than physician-obtained cervical specimens. ${ }^{1617}$ This was confirmed in our study that showed no statistical difference between self-collected and physician-collected specimens. It would be feasible for a large number of Cambodian women to self-collect swabs; the careHPV system, which is highly portable, could be used to rapidly test these specimens. In addition, the cost is relatively inexpensive at only approximately US $\$ 4$ per specimen.

The second component of this proposed strategy would be to perform DC on hrHPV+ women with a highly portable system such as the EVA system used in this study. Given the shortage of trained gynaecologists in Cambodia, DC can be performed by trained nurses or midwives. While previous studies regarding VIA have shown that physicians are more likely to detect CIN2+ than nurses, DC has several benefits over VIA that can help reduce the difference between nurses and physicians. ${ }^{24}{ }^{25}$ First, the images obtained (figure 2) have excellent resolution and can be easily magnified to visualise morphological feature consistent with high dysplasia such as mosaicism and punctation (figure 2). In our study, physicians were able to distinguish between CIN1 and CIN2+ lesions in all 30 patients who had confirmatory biopsies. Second, the DC images can be used for continued education of nurse/ midwives and for quality control. Lastly, the digital images can be sent, via a secure, cloud-based portal, for consultation in real time by an expert colposcopist anywhere in Cambodia or around the world.

The current WHO guidelines for low-resource countries such as Cambodia conditionally recommends using a strategy of 'screen with an HPV test followed by VIA and treat, over a strategy of screen with an HPV test followed by colposcopy (with or without biopsy) and treat'. ${ }^{26}$ The WHO guidelines recognise that their conditional recommendation is based on a 'very low quality of evidence'. The justification used in the guidelines to recommend VIA over colposcopy after HPV testing is that "there may be more resource implications with colposcopy due to increased training of providers, quality control, waiting time, and the potential for more women to be lost to follow-up'. ${ }^{26}$ 


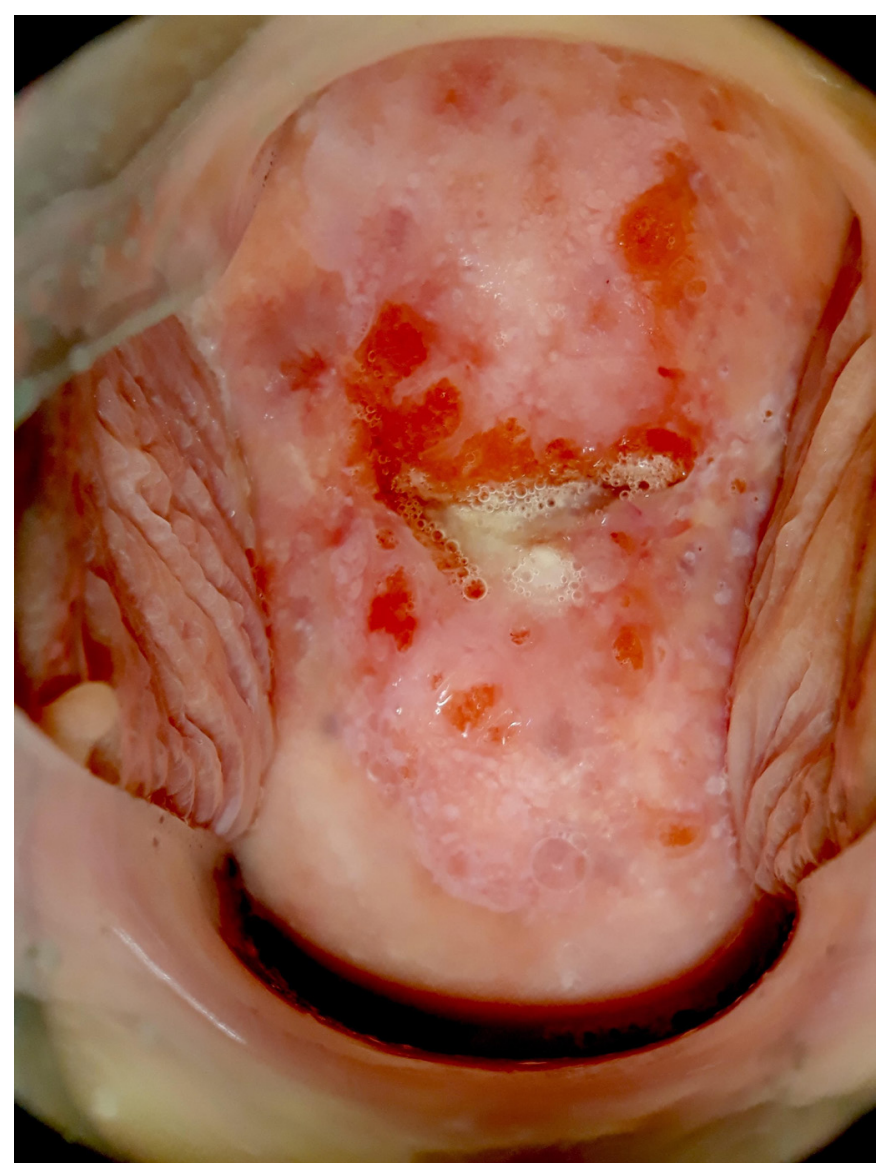

Figure 2 Cervical intraepithelial neoplasia 2 with mosaicism at 6 and 12 o'clock.

However, these recommendations were written prior to the development of a high-quality, portable, digital colposcope such as the EVA system, which does not have these limitations. In addition, the WHO guidelines recognise that there may be fewer CIN2+ recurrences with the HPV test followed by colposcopy without biopsy (3/1000 fewer) as well as fewer cervical cancers $(1 / 10000$ fewer with or without biopsy) and fewer deaths (6/100 000 fewer with or without biopsy) than with an HPV test followed by VIA. As such, given the new evidence put forth in our study, we suggest that the WHO guidelines might be modified in countries with the necessary resources to favour DC over VIA after HPV testing, if the results of our study are confirmed in subsequent large-scale studies.

A potential third component of a rapid, and cost-effective, screen-and-treat strategy might be to use the clinical impression derived from the DC images to determine immediate treatment. Specifically, women with suspected CIN1 lesions would be immediately treated with cryotherapy and those with suspected CIN2+ lesionswould have LEEP. Again, our proposed screening strategy differs slightly from current WHO guidelines that recommend using cryotherapy as the primary treatment modality in all screen-and-treat protocols that do not have diagnostic histology. ${ }^{26}$ The WHO recommends that for screen-andtreat programmes LEEP should only be used in lesions that are greater than $75 \%$ of the exocervix or when the lesion extends into the cervical os. In contrast, the WHO currently recommends either LEEP or cryotherapy in women with histologically confirmed CIN2+ butit acknowledges that the overall benefits may be greater with LEEP because it is probable there is less recurrence of dysplasia with LEEP as compared with cryotherapy. ${ }^{13}$ The reason we recommend using LEEP in suspected CIN2+ lesions in our screen-and-treat strategy (which does not have confirmatory pathology) is that the results of our study show that DC was as accurate as confirmatory histology in its distinguishing between CIN1 and CIN2+ in all 24 patients. If this finding is confirmed in additional large-scale studies, it would be worthwhile to incur the added cost of LEEP in suspected CIN2+ lesions because of the decreased risk of recurrence with LEEP in CIN2+ lesions. However, as the majority of CIN1 lesions will not progress to higher-grade lesions, and since cryotherapy is less resource intensive and can be performed by nurse or midwives, it should be used for suspected CIN1 lesions.

One limitation of this study is that the careHPV system is less sensitive than both the Hybrid Capture 2 system and HPV-PCR. Previous studies have shown that the sensitivity of careHPV system to detect CIN2+ lesion is $88.1 \%$. Since HPV-PCR was not performed on all specimens, we cannot give the exact prevalence of hrHPV in our patient population. An additional limitation of this study is that the results may not be applicable to women in more rural regions of Cambodia. While patients were recruited from both urban and rural settings for this study, there are ethnic groups, especially from the more mountainous regions of Cambodia, not well represented in our study population. Potentially, women from these ethnicities might not be as comfortable in self-swabbing as the women in our study.

Conclusion

Cost-effective, efficient and large-scale screening programmes must be developed in resource-poor countries such as Cambodia to screen hundreds of millions of women who have never been screened for cervical dysplasia and cancer. As such, screening strategies based on new technologies such as the careHPV system, which can rapidly and relatively inexpensively screen women for hrHPV, and the EVA digital colposcope, which can accurately (and without added cost) detect cervical dysplasia in women hrHPV+, need to be embraced. The results of this study will be used to propose a screening strategy to the Cambodian Ministry of Health.

\section{Author affiliations}

${ }^{1}$ Department of Gynecology, Sihanouk Hospital Center of Hope, Phnom Penh, Cambodia

${ }^{2}$ Obstetrics and Gynecology, The George Washington University School of Medicine and Health Sciences, Washington, District of Columbia, USA

${ }^{3}$ The Center for Vulvovaginal Disorders, Washington, District of Columbia, USA

${ }^{4}$ Yale University, New Haven, Connecticut, USA 
Contributors ST: development of the research protocol, data acquisition, interpretation of data, and preparation of manuscript. AG: development of the research protocol, data analysis, interpretation of data, and preparation of manuscript. LSG: development of the research protocol, interpretation of data, preparation of manuscript. VG: data analysis, preparation of manuscript. KL: data acquisition. CS: data acquisition.

Funding The Gynecologic Cancers Research Foundation, a Maryland, USA non-profit 501c3 corporation.

Competing interests Dr AG is on the Board of Directors of the Gynecologic Cancers Research Foundation. Advisory Board: Strategic Science and Technologies, Grunenthal, Ipsen, Amag. Research Funding: Strategic Science and Technologies, Grunenthal, Ipsen, Endoceutics, The Gynecologic Cancers Research Foundation, Cellular Medicine Association, ELeN.

Patient consent for publication Not required.

Ethics approval IRB approval was obtained from the National Committee on Health Research of the Cambodian Ministry of Health.

Provenance and peer review Not commissioned; externally peer reviewed.

Data sharing statement Extra data can be accessed via the Dryad data repository at http://datadryad.org/ with the doi:10.5061/dryad.jb4645r.

Open access This is an open access article distributed in accordance with the Creative Commons Attribution Non Commercial (CC BY-NC 4.0) license, which permits others to distribute, remix, adapt, build upon this work non-commercially, and license their derivative works on different terms, provided the original work is properly cited, appropriate credit is given, any changes made indicated, and the use is non-commercial. See: http://creativecommons.org/licenses/by-nc/4.0/.

\section{REFERENCES}

1. Ferlay J, Colombet M, Soerjomataram I, et al. Estimating the global cancer incidence and mortality in 2018: GLOBOCAN sources and methods. Int J Cancer 2018

2. Progress in Cervical Cancer Prevention: The CCA Report Card. 2015

3. Sovannara Thay SP, Hur C, Tramontano AC, et al. Prevalence of cervical dysplasia in HIV-positive and HIV-negative women in Phnom Penh, Cambodia. The Asian-Pacific Journal of Cancer Prevention. In Press. 2018.

4. Forman D, de Martel C, Lacey CJ, et al. Global burden of human papillomavirus and related diseases. Vaccine 2012;30(Suppl 5):F12-23.

5. de Martel C, Plummer M, Vignat J, et al. Worldwide burden of cancer attributable to HPV by site, country and HPV type. Int J Cancer 2017;141:664-70.

6. Touch S, Oh JK. Knowledge, attitudes, and practices toward cervical cancer prevention among women in Kampong Speu Province, Cambodia. BMC Cancer 2018;18:294.

7. Ferlay J, Soerjomataram I, Dikshit R, et al. Cancer incidence and mortality worldwide: sources, methods and major patterns in GLOBOCAN 2012. Int J Cancer 2015;136:E359-86.

8. Blumenthal PD, Mclntosh NACOGFIGO. Cervical cancer prevention in low-resource settings. Int J Gynaecol Obstet 2005;90:86-7.

9. Vun MC, Fujita M, Rathavy T, et al. Achieving universal access and moving towards elimination of new HIV infections in Cambodia. J Int AIDS Soc 2014;17:18905.

10. Massad LS, Xie X, D'Souza G, et al. Incidence of cervical precancers among HIV-seropositive women. Am J Obstet Gynecol 2015;212:606.e1-8.
11. Frisch M, Biggar RJ, Goedert JJ. Human papillomavirus-associated cancers in patients with human immunodeficiency virus infection and acquired immunodeficiency syndrome. J Natl Cancer Inst 2000;92:1500-10.

12. Forhan SE, Godfrey CC, Watts $\mathrm{DH}$, et al. A systematic review of the effects of visual inspection with acetic acid, cryotherapy, and loop electrosurgical excision procedures for cervical dysplasia in HIV-infected women in low- and middle-income countries. J Acquir Immune Defic Syndr 2015;68(Suppl 3):S350-6.

13. Kelly $\mathrm{H}$, Weiss $\mathrm{HA}$, Benavente $\mathrm{Y}$, et al. Association of antiretrovira therapy with high-risk human papillomavirus, cervical intraepithelial neoplasia, and invasive cervical cancer in women living with HIV: a systematic review and meta-analysis. Lancet HIV 2018;5:e45-58.

14. WHO Guidelines Approved by the Guidelines Review Committee. WHO Guidelines: Use of Cryotherapy for Cervical Intraepithelial Neoplasia. Geneva: World Health Organization World Health Organization, 2011.

15. WHO Guidelines Approved by the Guidelines Review Committee. WHO Guidelines for Treatment of Cervical Intraepithelial Neoplasia 2-3 and Adenocarcinoma in situ: Cryotherapy, Large Loop Excision of the Transformation Zone, and Cold Knife Conization. Geneva: World Health Organization, 2014.

16. Blumenthal PD, Lauterbach M, Sellors JW, et al. Training for cervical cancer prevention programs in low-resource settings: focus on visual inspection with acetic acid and cryotherapy. Int J Gynaecol Obstet 2005;89(Suppl 2):S30-7.

17. Jeronimo J, Bansil P, Lim J, et al. A multicountry evaluation of careHPV testing, visual inspection with acetic acid, and papanicolaou testing for the detection of cervical cancer. Int $J$ Gynecol Cancer 2014;24:576-85.

18. Arbyn M, Verdoodt F, Snijders PJ, et al. Accuracy of human papillomavirus testing on self-collected versus clinician-collected samples: a meta-analysis. Lancet Oncol 2014;15:172-83.

19. Koliopoulos G, Nyaga VN, Santesso N, et al. Cytology versus HPV testing for cervical cancer screening in the general population. Cochrane Database Syst Rev 2017;8:Cd008587.

20. Spitzer M. The Era of "Digital Colposcopy" Will Be Here Soon. J Low Genit Tract Dis 2015;19:273-4.

21. Liu AH, Gold MA, Schiffman M, et al. Comparison of colposcopic impression based on live colposcopy and evaluation of static digital images. J Low Genit Tract Dis 2016;20:154-61.

22. Hermens M, Ebisch RM, Galaal K, et al. Alternative colposcopy techniques: a systematic review and meta-analysis. Obstet Gynecol 2016;128:795-803.

23. Organization WH. Human resources for health country profiles. Cambodia, 2014.

24. Raifu AO, El-Zein M, Sangwa-Lugoma G, et al. Determinants of cervical cancer screening accuracy for Visual Inspection with Acetic Acid (VIA) and Lugol's lodine (VILI) performed by nurse and physician. PLoS One 2017;12:e0170631.

25. Sherigar B, Dalal A, Durdi G, et al. Cervical cancer screening by visual inspection with acetic acid-interobserver variability between nurse and physician. Asian Pac J Cancer Prev 2010;11:323-6.

26. WHO Guidelines Approved by the Guidelines Review Committee. WHO Guidelines for Screening and Treatment of Precancerous Lesions for Cervical Cancer Prevention. Geneva: World Health Organization, 2013. 